\title{
A novel bi-alleleic DDX41 mutations in B-cell lymphoblastic leukemia: case report
}

\author{
Woo Yong Shin ${ }^{1 \dagger}$, Seug Yun Yoon ${ }^{2 \dagger}$, Rojin Park', Jung-Ah Kim ${ }^{1}$, Ho Hyun Song ${ }^{3}$, Hae In Bang ${ }^{1}$, \\ Jong-Ho Won ${ }^{2}$ and Jieun Kim ${ }^{1 *}$ (D)
}

\begin{abstract}
Background: The germline mutations of DDX41, also known as DEAD box RNA helicase 41 , have been found in about 1.5\% of myeloid neoplasms (MNs). Development of MDS/AML is relatively common in germline DDX41 mutations. However, a variety of hematological malignancies (HMs) have been reported.

Case presentation: We report a novel case of bi-alleleic DDX41 mutations in B-cell lymphoblastic leukemia (B-ALL), with unusual location of DDX41 mutations. The gene expression profile (GEP) of Ph + B-ALL with bi-alleleic DDX41 mutations showed heterogeneously transitional GEP and altered gene expression levels of genes involved in the process essential for red blood cells and myeloid cell differentiation were noted.

Conclusions: We report that DDX41 mutations are unusual but can be an underlying event in Ph + B-ALL and screening DDX41 mutations can be also informative for patients awaiting for haploidentical stem cell transplantation and choosing the therapy.
\end{abstract}

Keywords: DDX41 germline mutation, B-cell lymphoblastic leukemia, Gene expression, Case report

\section{Background}

The awareness of the hereditary basis for hematologic malignancies (HMs) is increasing, such germline mutations are found in $4.4 \%$ to $18 \%$ of HM patients, depending on population [1]. The germline mutations of $D D X 41$, also known as DEAD box RNA helicase 41, have been found in about $1.5 \%$ of myeloid neoplasms (MNs) [2]. Families with DDX41 mutations display an autosomal dominant inheritance, with a clinical picture dominated by late onset of either myelodysplastic syndrome (MDS) or acute myeloid leukemia (AML) [3].

Development of MDS/AML is relatively common in germline DDX41 mutations. However, a variety of hematological malignancies (HMs) have been reported,

\footnotetext{
*Correspondence: jkim1220@gmail.com

†Woo Yong Shin and Seug Yun Yoon contributed equally to this work

${ }^{1}$ Department of Laboratory Medicine, Soonchunhyang University Seoul

Hospital, Soonchunhyang University College of Medicine, Seoul, Korea

Full list of author information is available at the end of the article
}

including rare cases of chronic myeloid leukemia (CML), and lymphoma, which means that mutations in DDX41 cannot be attributed to a specific malignant disorder [4]. Therefore, an association between the types of DDX41 mutations, accumulation of secondary mutations, or type of leukemia may pertain to its role in the leukemogenesis.

The processes by which the DDX41 mutation contributes to the oncogenesis that leads to myeloid neoplasms (MNs) have been investigated, but the underlying molecular pathogenesis of DDX41 mutations in B lymphoblastic leukemia (B-ALL) has not been revealed. Genetic expression profiling (GEP) has previously proven useful in B-ALL for identifying signatures of oncogenes, with the recognition of novel subgroups, as well as with outcome [5]. Therefore, we adopted GEP of a novel case of B-ALL with $\mathrm{t}(9 ; 22) B C R-A B L 1$ harboring $D D X 41$ germline and somatic mutations, to uncover the contribution of DDX41 to leukemogenesis. In addition, we compared the GEP of the present case with other relevant samples, including B-ALL with $\mathrm{t}(9 ; 22) B C R-A B L 1$ 
and AML with bi-alleleic DDX41 mutations, providing cluster analysis and thereby taking a step closer to understanding of the underlying mechanisms.

\section{Case presentation}

\section{Case description}

A 48-year-old man with a past medical history of hypertension, hyperlipidemia, and asthma presented with fatigue, and night sweats, and there were no palpable lymph nodes in his physical examination. Initial complete blood- cell counts included a hemoglobin $(\mathrm{Hb})$ of $12.5 \mathrm{~g} / \mathrm{dL}$, white blood- cell (WBC) count of $22.1 \times 10^{9} / \mathrm{L}$ with $67 \%$ blasts on peripheral blood smear, and a platelets count of $91 \times 10^{9} / \mathrm{L}$. In the bone- marrow $(\mathrm{BM})$ aspiration, blasts accounted for $88.1 \%$ of ANCs, which were positive for CD9, CD10, CD13, CD19, CD20, CD34, CD38, CD58, CD66c, CD123, HLA-DR, cCD79a, and $\mathrm{TdT}$ on flow cytometry. The karyotype was revealed as 46,XY,t(9;22)(q34;q11.2)[10]/47,idem, + der(22)t(9;22)[2] by chromosomal tests. $B C R-A B L 1$ fusion was detected by FISH analysis and confirmed as a major transcript (b3a2). There was no specific familial history for any hematologic malignancies.

\section{Genomic sequencing and microarray analysis of expression profiles in samples}

IKZF1 exon 4 6 somatic deletion was detected in the copy number variant analysis of the next- generation sequencing (NGS). Aside from the somatic $A B L 1$ mutation (c.688C $>\mathrm{T}$, p.Pro230Ser), we found two novel DDX41 mutations, c.639delC, p.Thr214Profs" 8 and c.259C > T, p.Leu87Phe, and confirmed the frameshift variant of DDX41 being of germline origin by Sanger sequencing of skin fibroblast (Fig. 1A, B) (see Additional file 4). Both mutations were not reported in gnomAD, 1000 Genomes, and HGVD database. Sorting Intolerant From Tolerant (SIFT) calculated that p.Leu87Phe has deleterious effect and PolyPhen-2 predicted to be probably damaging.

Genetic analysis was performed on five patients, including the present study ( $\left.\mathrm{Ph}+\mathrm{B}-\mathrm{ALL}{ }^{I K Z F 1+\mid D D X 41 d m}\right)$, two cases of Ph $+\mathrm{B}$-ALL accompanying IKZF1 deletion without DDX41 mutation ( $\left.\mathrm{Ph}+\mathrm{B}-\mathrm{ALL}^{I K Z F 1+/ D D X 41-}\right)$, one case of AML with double $D D X 41$ mutation $\left(\mathrm{AML}^{D D X 41}\right.$ $d m$ ), and one case of normal BM, whom were examined for HM at Soonchunhyang University Seoul Hospital, South Korea, from November 2018 to May 2020. This study was approved by the Institutional Review Board of Soonchunhyang University Seoul Hospital (IRB no. 2021-01-003). The fresh BM, whole blood (WB), and skin fibroblast specimens were stored at $-80{ }^{\circ} \mathrm{C}$ before genome sequencing and GEP.
Briefly, genomic DNA were extracted from proband's skin fibroblast, WB, and BM samples at initial diagnosis using a QIAamp DNA Blood Mini Kit (Qiagen, MD, USA) according to standard procedures. Targeted NGS with a hematologic malignancy comprehensive panel (Celemics, Seoul, South Korea), which examines 85 hematologic malignancy- associated genes (see Additional file 1) were performed in all samples. We confirmed sequence mutations and exonal deletions by Sanger sequencing and multiplex ligation-dependent probe amplification (MLPA) (SALSA MLPA P335-C1 ALL-IKZF1 probemix, MRC Holland, Amsterdam, Holland), respectively.

To determine whether two mutations in different regions of the $D D X 41$ of the patient were in different alleles, the cDNA including region of interest was amplified with the following primers (Forward, 5'-gaggaagagcagcaggacag-3'; Reverse, 5'-tcatgtcacggccagataga- $\left.3^{\prime}\right)$. The PCR product was then cloned into the TA-cloning vector (Topcloner TA kit; Enzynomics, Daejeon, Korea) and ten clones were sequenced which included the corresponding regions of the DDX41.

RNA was extracted from proband's BM samples at initial diagnosis using an RNeasy Micro kit (Qiagen) and then evaluated with an Agilent 2100 Bioanalyzer (Agilent Technologies, Santa Clara, USA) for RNA integrity. We obtained a total of $3.5 \mu \mathrm{g}$ of cDNA after amplification with a GeneChip ${ }^{\mathrm{TM}}$ WT Pico Kit (Affymetrix, CA, USA) and processed it for GEP. GEPs were generated using the GeneChip Human Gene 2.0 ST Array (Affymetrix). A robust multi-average (RMA) method implemented in Affymetrix ${ }^{\circledR}$ Power Tools (APT) was used for data summarization and normalization. The results were exported to gene-level RMA analysis and differentially expressed gene (DEG) analysis was performed. Statistical significance of the expression data was determined using fold change. Gene-enrichment and functional annotation analysis for a significant probe list was performed using Gene Ontology (http://geneontology.org) and KEGG (Kyoto Encyclopedia of Genes and Genomes, http://kegg. jp). All data analysis and visualization of differentially expressed genes was conducted using $\mathrm{R}$ 3.3.2 (The $\mathrm{R}$ Foundation for Statistical Computing, Vienna, Austria).

We compared the differentially expressed genes of $\mathrm{Ph}+\mathrm{B}-\mathrm{ALL}^{I K Z F 1+/ D D X 41 d m}$ with two cases of $\mathrm{Ph}+\mathrm{B}-\mathrm{ALL} \mathrm{L}^{I K Z F 1+\mid D D X 41-}$ in order to elucidate the role of DDX41 in leukemogenesis. Assessment of differential expression between samples was conducted employing linear models for microarrays in $\mathrm{R}$, and genes with fold change $\geq 3$ and $p<0.05$ were to be considered significant. For a DEG set, hierarchical cluster analysis was done in all samples in order to assess the degree of relatedness; 


\section{$\bigcirc$ Present case $\bigcirc$ Previous reports}

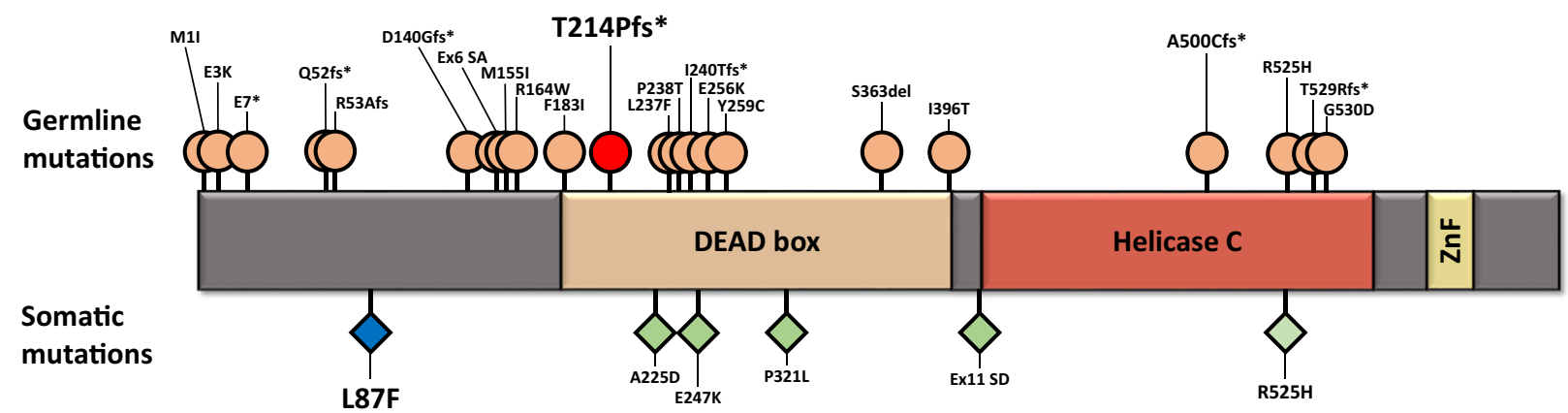

A

SA splice acceptor, SD splice donor

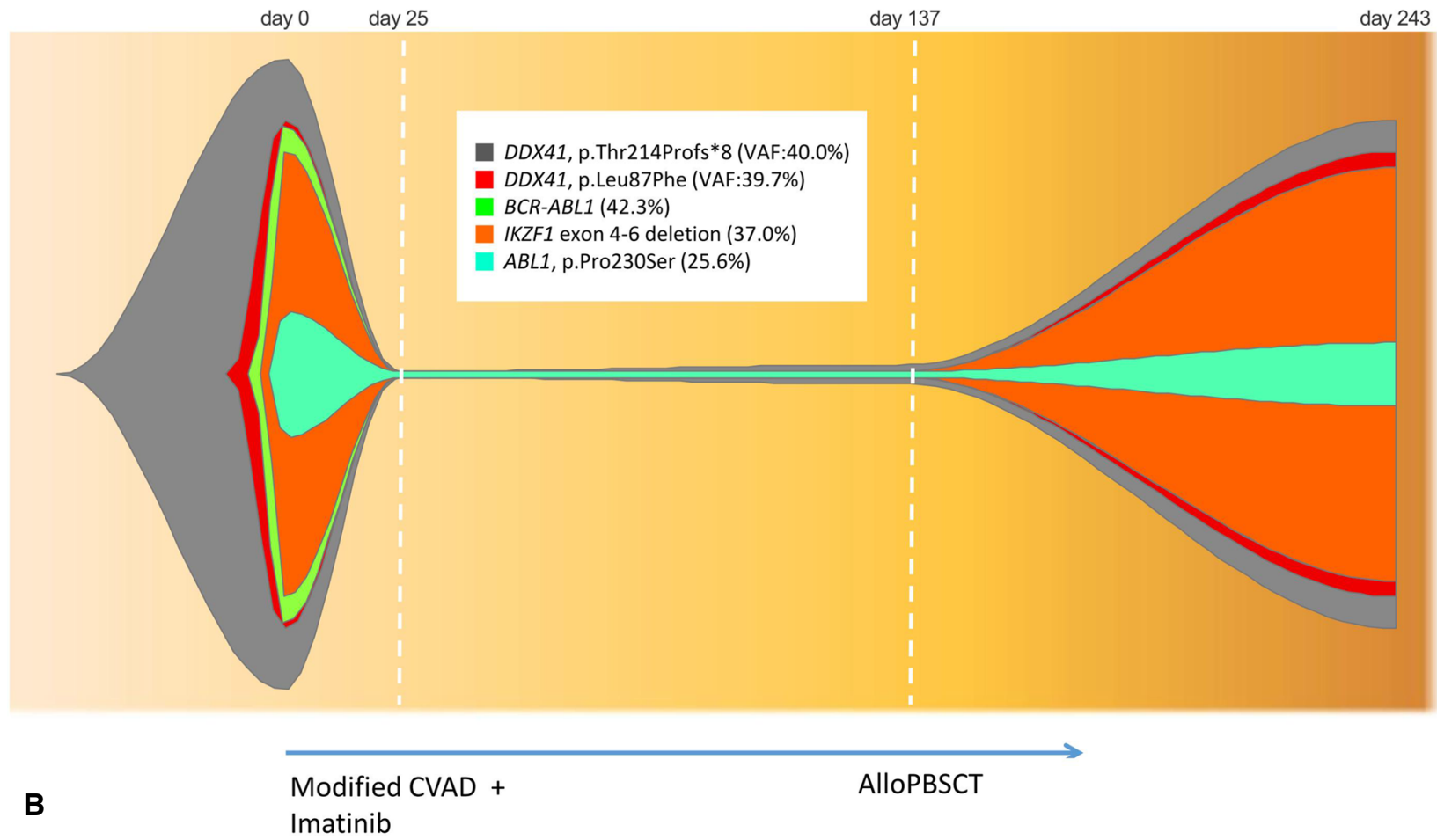

Fig. 1 A Schematic representation of the reported germline and somatic DDX41 mutations in hematologic malignancies and present study $\mathbf{B}$ Clonal architectures of our case series during the course of treatment. Variant allele frequency (VAF) for each mutation is indicated

and samples were clustered using complete linkage and Euclidean distance as a measure of similarity.

\section{Results}

The patient visited this medical center outpatient for 17 years before being diagnosed, and except for April 2015, when neutrophils were elevated due to pneumonia, WBC count was $6.1-10.0 \times 10^{9} / \mathrm{L}$ and differential count was normal. The last visit was 3 years before diagnosis and there was no CML related morphologic evidence such as basophilia and myeloid proliferation at diagnosis. Therefore, it was determined that there would be no underlying disease such as CML, the patient was diagnosed as B-ALL with $\mathrm{t}(9 ; 22)(\mathrm{q} 34.1 ; \mathrm{q} 11.2)$ and received induction chemotherapy of modified Hyper-CVAD regimen (cyclophosphamide, vincristine, adriamycin, dexamethasone and pegylated asparaginase) with imatinib. After a month of induction therapy, blasts were decreased 
to $0.1 \%$ of ANCs in the follow-up BM exam, and $B C R$ $A B L 1$ transcripts were decreased to $<0.0004 \%$ on the international scale. The patient received allogenic peripheral blood stem cell transplantation (SCT) from a sibling donor without germline DDX41 mutation after consolidation chemotherapy with high-dose cytarabine and mitoxantrone. However, B-ALL relapsed nine months later, with an increased WBC count of $85.2 \times 10^{9} / \mathrm{L}$ with $81 \%$ blasts on peripheral blood.

Clinical features and genetic alterations detected in genomic DNA sequencing of all samples are shown (see Additional file 2); and the clonal architecture of the present case during the course of treatment are is depicted in Fig. 1. Two mutations, c.639delC and c. $259 \mathrm{C}>\mathrm{T}$, were confirmed to be present in different alleles. Of 53,617 transcripts represented by the microarray, 409 were differentially over- or under-expressed

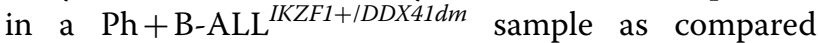
to $\mathrm{Ph}+\mathrm{B}-\mathrm{ALL}{ }^{I K Z F 1+/ D D X 41-}$ samples. Among those 409 transcripts, 233 were expressed more abundantly and 176 less abundantly in $\mathrm{Ph}+\mathrm{B}-\mathrm{ALL}{ }^{I K Z F 1+/ D D X 41 d m}$ (Fig. 2A). A list of the 409 dysregulated transcripts for $\mathrm{Ph}+\mathrm{B}-\mathrm{ALL}^{I K Z F 1+/ D D X 41 d m}$ is shown in more detail (see Additional file 3). To summarize, among DEGs, the expression of DDX41 in case sample (Ph + B-ALL ${ }^{I K Z F 1+1}$ $D D X 41 d m$ ) was characterized by high-level expression of a set of genes involved in p53 signaling pathway when compared to $\mathrm{Ph}+\mathrm{B}-\mathrm{ALL}{ }^{I K Z F 1+/ D D X 41-}$ samples, whereas $B$ cell receptor signaling pathway, PI3K-Akt signaling pathway, and NF-kappa B signaling pathway were differentially expressed compared to AML ${ }^{D D X 41 d m}$.

To elucidate the biological significance of differentially expressed genes in $\mathrm{Ph}+\mathrm{B}-\mathrm{ALL}{ }^{I K Z F 1+/ D D X 41 d m}$, gene ontology (GO) analysis of the whole transcriptome was performed. Of the 3,214 functional categories examined, the top DEGs are presented in Fig. 2B. GO analysis revealed that significant categories for expressed genes seem to have a strong correlation with the process essential for red blood cells and myeloid cells. Pathway enrichment revealed that there were 11 significant pathways were enriched in $\mathrm{Ph}+\mathrm{B}-\mathrm{ALL}^{I K Z F 1+/ D D X 41 d m}$ compared to $\mathrm{Ph}+\mathrm{B}-\mathrm{ALL}{ }^{I K Z F 1+/ D D X 41-}$, and one of the most significant pathways was transcriptional misregulation in cancer (hs05202) genes $(p<0.001)$, which contained common cancer-related genes, such as HIST1H3G, SUPT3H, HIST1H3I, BCL2L1, WT1, HIST1H3A, CD86, CDK14, CSF1R, and PROM1. Detailed analysis of the dysregulated genes revealed several candidates linked to relevant signaling pathways in $\mathrm{Ph}+\mathrm{B}-\mathrm{ALL} \mathrm{L}^{I K Z F 1+/ D D X 41 d m}$, which may represent pathogenetically relevant genes. Genes associated with proliferation, and cell survival (BCL2L1), and tumor- cell growth (WT1) pathways for development of cancer were overexpressed, whereas molecules relevant to differentiation resistance (CSF1R) were underexpressed. Based on hierarchical cluster analysis, $\mathrm{Ph}+\mathrm{B}-\mathrm{ALL}{ }^{I K Z F 1+/ D D X 41 d m}$ can be distinguished from $\mathrm{Ph}+\mathrm{B}-\mathrm{ALL}{ }^{I K Z F 1+/ D D X 41-}$ and AML ${ }^{D D X 41 d m}$ and that the

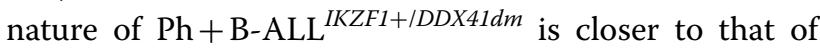
$\mathrm{Ph}+\mathrm{B}-\mathrm{ALL}{ }^{I K Z F 1+/ D D X 41-}$ than to that of AML ${ }^{D D X 41 d m}$, as shown in heatmap analysis (Fig. 3).

\section{Discussion and conclusions}

Inherited DDX41 mutations are always heterozygous and usually in frame-shift mutations, indicating a potential loss-of-function (LOF). Approximately half of MN patients with inherited DDX41 mutations acquire a second-hit, often $\mathrm{R} 525 \mathrm{H}$, in the healthy DDX41 allele in their disease clones [3]. To date, in all reported bi-allelic DDX41 mutated HM cases, germline mutations rather than somatic mutations occurred relatively at the forefront of, except for an MDS case with germline R369G and somatic $54 *[6]$. In our case, the location of $D D X 41$ mutations differed from that in previous reports, where somatic and germline mutations occurred in the N-terminal domain and DEAD box domain, respectively.

Since $B C R-A B L 1$ translocation alone is insufficient for malignant transformation, it is known that various complex additional mutations are required for $\mathrm{Ph}+\mathrm{B}$-ALL development [7]. Over 70\% of Ph + B-ALL patients harbor IKZF1 LOF [7], however, to the best of our knowledge, concomitant $D D X 41$ mutations have never been reported. Furthermore, $5 \mathrm{q}$ deletion is also rarely observed in ALL, which we hypothesize to have resembled the GEP of our case, that deduction of DDX41 mutation on lymphoid malignancy is challenging [8]. The previously reported cases have shown, DDX41 has been shown to be a cytoplasmic DNA sensor in dendritic cells and to have a documented role in the innate immune response [9]. Therefore, dysregulation of such responses may be an initiator of disorders and may be linked to lymphoid malignancy.

The interesting feature of our case was the concomitant mutation on $A B L 1$ (c.688C $>$ T, p.Pro230Ser), which resides in the $\mathrm{SH} 2$-kinase linker domain of $A B L 1$ and is seldom observed in a $B C R-A B L 1$ transcript [10]. Association of $D D X 41$ mutations with this finding is uncertain,; however, $D D X 41$ mutations are largely mutually exclusive to with splice- factor mutations [11]. The loss of tumor suppressor function because of altered pre-mRNA splicing and RNA processing is another aspect of somatic DDX41 mutations [2]. In our case, mutations were not detected in genes of the splice-factor family, and an explanation for this observation remains elusive.

We found altered gene expression levels of genes involved in the process essential for red blood cells, which is consistent with previous observations that 


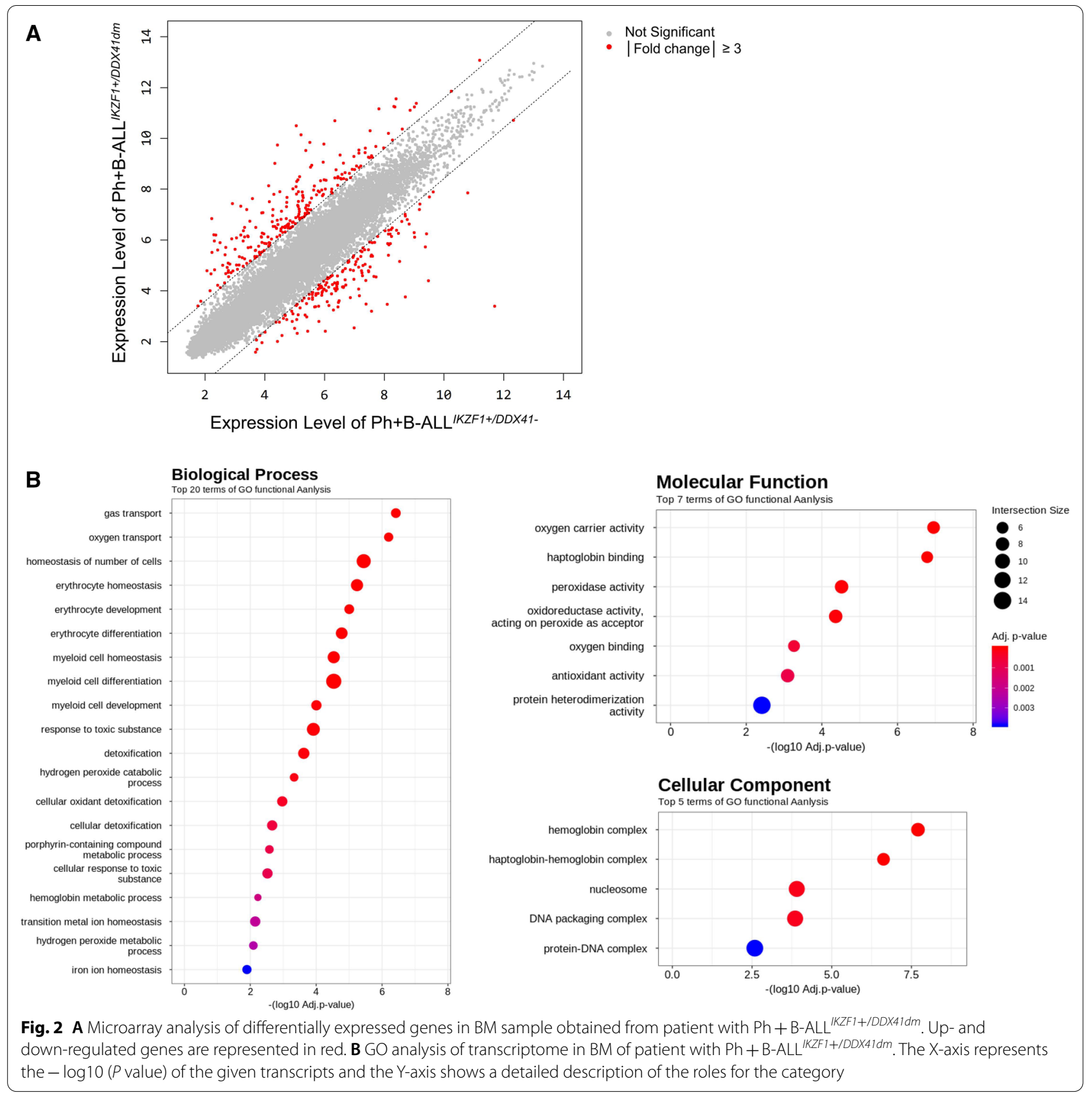

DDX41 mutations, including LOF, can affect erythroid differentiation [12]. For myeloid cell differentiation, $\mathrm{Ph}+\mathrm{B}-\mathrm{ALL}^{I K Z F 1+/ D D X 41 d m}$ had significantly altered levels of expression compared to Ph + B-ALL ${ }^{I K Z F 1+/ D D X 41-}$. However, the contribution of $D D X 41$ mutations, including gain-of-function, to developing myeloid malignancy is not fully understood, but is presumed to be involved in the pathogenesis of a certain subset of such AML cases [13].
Based on case reports and retrospective analysis, lenalidomide has been suggested as an effective treatment strategy for myeloid malignancies with $D D X 41$ mutations [14]. The patient in our study has undergone a combination of modified CVAD and imatinib, which is front-line therapy for adult $\mathrm{Ph}+\mathrm{B}$-ALL [15]. Because of short follow-up time, whether the efficacy of lenalidomide might have been beneficial for this patient is could not be addressed. However, the genetic testing 


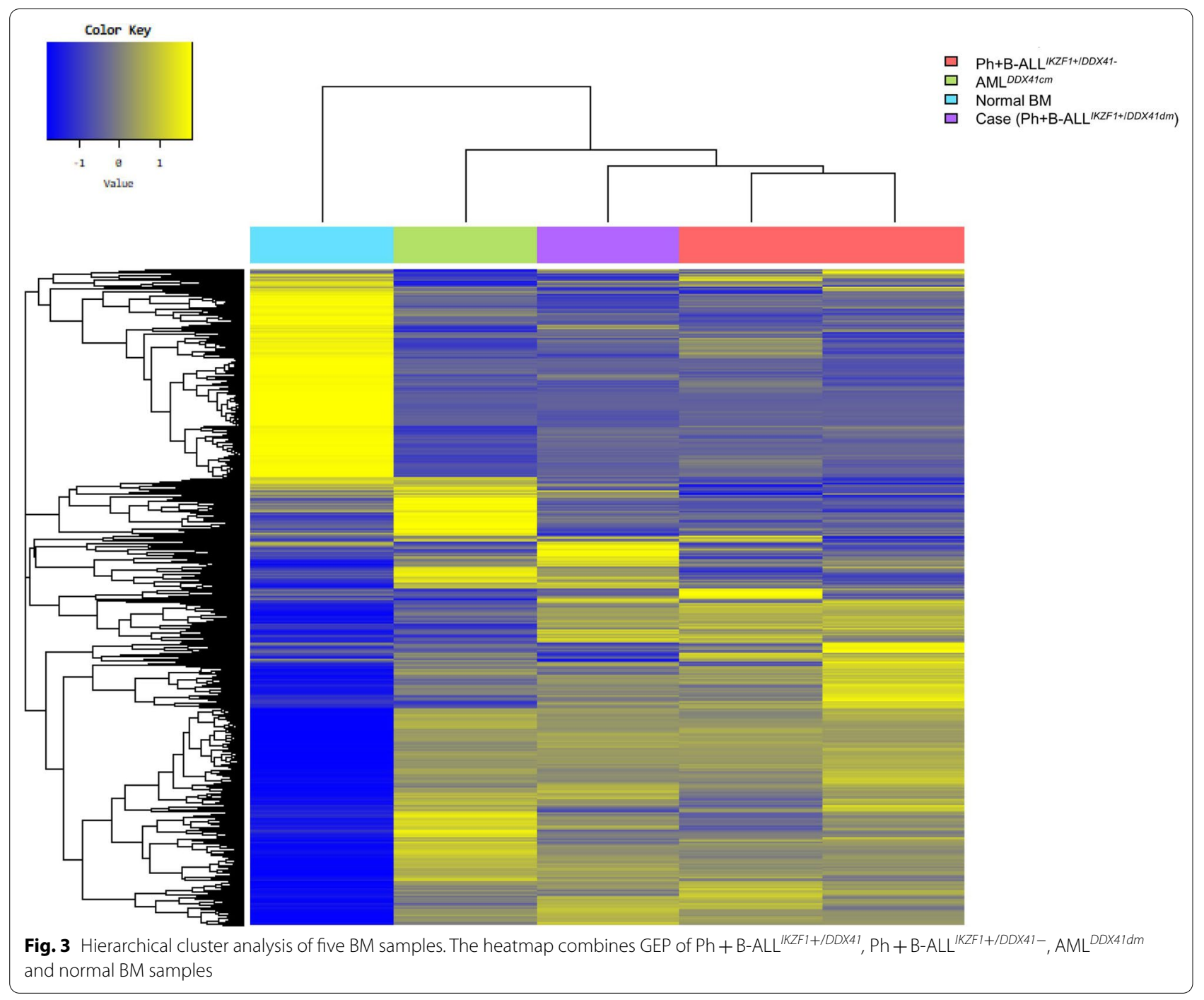

for $D D X 41$ to find an optimal family-member donor was performed done in a timely manner.

Herein, we report that $D D X 41$ mutations are unusual but can be an underlying event in $\mathrm{Ph}+\mathrm{B}$-ALL, although the causative link between $D D X 41$ variants and B-ALL is yet to be established; however, it shows heterogeneously transitional GEP of both Ph + B-ALL and AML with DDX41 mutations. Screening DDX41 mutations can be also informative for patients awaiting for haploidentical SCT and choosing the therapy.

\section{Abbreviations}

B-ALL: B lymphoblastic leukemia; GEP: Gene expression profiling; HM: Hematologic malignancy; BM: Bone marrow; WB: Whole blood; SCT: Stem cell transplantation; LOF: Loss-of-function.

\section{Supplementary Information}

The online version contains supplementary material available at https://doi. org/10.1186/s12920-022-01191-2.

Additional file 1: The list of 85 hematologic malignancy associated genes included in customized NGS panel used this study

Additional file 2: Genetic alterations of the case series of this study

Additional file 3: A list of the 409 dysregulated transcripts for $\mathrm{Ph}+\mathrm{B}-\mathrm{ALL}$ IKZFI+/DDX41dm

Additional file 4: Description: Supplementary figure. (A) c.639delC, p.Thr214Profs $* 8$ mutation of the patient's bone marrow sample (B) Same mutation found in the patient's skin fibroblasts (C) C.259C > T, p.Leu87Phe mutation of the patient's bone marrow sample (D) The patient's frameshift mutation confirmed by Sanger sequencing for bone marrow (E) Sanger sequencing for the sibling's peripheral blood revealed presence of c.639delC. 


\section{Acknowledgements \\ Not applicable.}

\section{Authors' contributions}

WYS and SYY participated in the writing of the paper. RP participated in design of the work. JAK participated in primary data acquisition and clinical analysis. HHS participated in an additional data acquisition and analysis. HIB and JHW participated in substantively revised the paper. JK participated in research design and acquisition of financial support. All authors have approved the submitted version and agreed both to be personally accountable for the author's own contributions and to ensure that questions related to the accuracy or integrity of any part of the work.

\section{Funding}

This study was supported by a grant (NRF-2020R1C1C1008535) of the National Research Foundation of Korea and the Soonchunhyang University Research Fund, Korea. The funding bodies played no role in the design of the study and collection, analysis, and interpretation of data and in writing the manuscript.

\section{Availability of data and materials}

The datasets analyzed in the current study are available in the NCBI's Gene Expression Omnibus (GEO) with accession number GSE1 96107 (https://www. ncbi.nlm.nih.gov/geo/query/acc.cgi?acc=GSE196107) for gene expression and DDBJ Sequenced Read Archive (https://ddbj.nig.ac.jp/search), under the Bioproject accession number PRJDB13084 (Biosample: SAMD00443631SAMD00443638) for genomic sequencing, respectively.

\section{Declarations}

\section{Ethics approval and consent to participate}

The study was approved by the Institutional Review Board (IRB) of Soonchunhyang University Seoul Hospital (IRB no. 2021-01-003) for the retrospective chart reviews and studies of existing preserved specimens all collected from subjects agreed and signed the informed consent.

\section{Consent for publication}

Written informed consent was obtained from all of the participants for publication of this case report and all materials. These materials include clinical details, characteristic phenotypic information, genetic testing results and other related data used in this report. A copy of the written consent is available for review by the Editor of this journal.

\section{Competing interests}

The authors declare that they have no competing interests.

\section{Author details}

'Department of Laboratory Medicine, Soonchunhyang University Seoul Hospital, Soonchunhyang University College of Medicine, Seoul, Korea. ${ }^{2}$ Division of Hematology and Oncology, Department of Internal Medicine, Soonchunhyang University Seoul Hospital, Soonchunhyang University College of Medicine, Seoul, Korea. ${ }^{3}$ Department of Interdisciplinary Program in Biomedical Science, Graduate School, Soonchunhyang University, Asan, Chungcheongnam-do, Korea.

Received: 23 September 2021 Accepted: 22 February 2022

Published online: 04 March 2022

\section{References}

1. Furutani E, Shimamura A. Germline genetic predisposition to hematologic malignancy. J Clin Oncol. 2017;35:1018-28.

2. Polprasert C, Schulze I, Sekeres MA, Makishima H, Przychodzen B, Hosono $\mathrm{N}$, et al. Inherited and somatic defects in DDX41 in myeloid neoplasms. Cancer Cell. 2015;27:658-70.

3. Cheah JJC, Hahn CN, Hiwase DK, Scott HS, Brown AL. Myeloid neoplasms with germline DDX41 mutation. Int J Hematol. 2017:106:163-74.
4. Swerdlow SH, Campo E, Pileri SA, Harris NL, Stein H, Siebert R, et al. The 2016 revision of the World Health Organization classification of lymphoid neoplasms. Blood. 2016;127:2375-90.

5. Chiaretti S, Messina M, Tavolaro S, Zardo G, Elia L, Vitale A, et al. Gene expression profiling identifies a subset of adult T-cell acute lymphoblastic leukemia with myeloid-like gene features and over-expression of miR223. Haematologica. 2010;95:1114-21.

6. Bannon SA, Routbort MJ, Montalban-Bravo G, Mehta RS, Jelloul FZ, Takahashi K, et al. Next-generation sequencing of DDX41 in myeloid neoplasms leads to increased detection of germline alterations. Front. Oncol. 2021;10.

7. Komorowski L, Fidyt K, Patkowska E, Firczuk M. Philadelphia chromosome-positive leukemia in the lymphoid lineage-similarities and differences with the myeloid lineage and specific vulnerabilities. Int J Mol Sci. 2020;21:5776.

8. Adriana Zamecnikova SaB. del(5q) in acute lymphoblastic leukemia (ALL). Atlas Genet Cytogenet Oncol Haematol. 2020;24:5

9. Lewinsohn M, Brown AL, Weinel LM, Phung C, Rafidi G, Lee MK, et al. Novel germ line DDX41 mutations define families with a lower age of MDS/AML onset and lymphoid malignancies. Blood. 2016;127:1017-23.

10. Khorashad JS, Kelley TW, Szankasi P, Mason CC, Soverini S, Adrian LT, et al. BCR-ABL1 compound mutations in tyrosine kinase inhibitor-resistant CML: frequency and clonal relationships. Blood. 2013;121:489-98.

11. Seiler M, Peng S, Agrawal AA, Palacino J, Teng T, Zhu P, et al. Somatic mutational landscape of splicing factor genes and their functional consequences across 33 cancer types. Cell Rep. 2018;23:282-96.e4.

12. Venugopal P, Cheah JJC, Eshraghi L, Shahrin NH, Homan C, Feng J, et al. An integrative genomic approach to examine mutations and biological pathways associated with hematological malignancy development in DDX41 mutated families. Blood. 2019;134:2686.

13. Kadono M, Kanai A, Nagamachi A, Shinriki S, Kawata J, Iwato K, et al. Biological implications of somatic DDX41 p. R525H mutation in acute myeloid leukemia. Exp Hematol. 2016:44:745-54.e4.

14. Abou Dalle I, Kantarjian H, Bannon SA, Kanagal-Shamanna R, Routbort M, Patel KP, et al. Successful lenalidomide treatment in high risk myelodysplastic syndrome with germline DDX41 mutation. Am J Hematol. 2020;95:227-9.

15. Thomas DA, Faderl S, Cortes J, O'Brien S, Giles FJ, Kornblau SM, et al. Treatment of Philadelphia chromosome-positive acute lymphocytic leukemia with hyper-CVAD and imatinib mesylate. Blood. 2004;103:4396-407.

\section{Publisher's Note}

Springer Nature remains neutral with regard to jurisdictional claims in published maps and institutional affiliations.

Ready to submit your research? Choose BMC and benefit from

- fast, convenient online submission

- thorough peer review by experienced researchers in your field

- rapid publication on acceptance

- support for research data, including large and complex data types

- gold Open Access which fosters wider collaboration and increased citations

- maximum visibility for your research: over $100 \mathrm{M}$ website views per year

At BMC, research is always in progress.

Learn more biomedcentral.com/submissions 